\title{
Biologics in the management of ulcerative colitis - comparative safety and efficacy of TNF- $\alpha$ antagonists
}

This article was published in the following Dove Press journal:

Therapeutics and Clinical Risk Management

5 January 2015

Number of times this article has been viewed

\author{
Rebecca Fausel' \\ Anita Afzali ${ }^{1,2}$ \\ 'Division of Gastroenterology, \\ Department of Medicine, University \\ of Washington, Seattle, WA, USA; \\ ${ }^{2}$ Inflammatory Bowel Disease \\ Program, UW Medicine - Harborview \\ Medical Center, Seattle, WA, USA
}

\begin{abstract}
Ulcerative colitis can cause debilitating symptoms and complications such as colonic strictures, colonic dysplasia, colorectal cancer, and toxic megacolon or perforation. Goals of treatment in ulcerative colitis include resolution of gastrointestinal symptoms, healing of colonic mucosa, and prevention of disease complications. Our treatment armamentarium has expanded dramatically over the past 10 years, and we now have multiple biologic agents approved for the treatment of moderate-severe disease, in addition to conventional therapies such as 5-aminosalicylates, thiopurines, and corticosteroids. In this review, we will provide a detailed discussion of the three tumor necrosis factor-alpha (TNF- $\alpha$ ) inhibitors currently approved for treatment of ulcerative colitis: infliximab, adalimumab, and golimumab. All three agents are effective for inducing and maintaining clinical response and remission in patients with ulcerative colitis, and they have comparable safety profiles. There are no head-to-head trials comparing their efficacy, and the choice of agent is most often based on insurance coverage, route of administration, and patient preference. Combination therapy with an immunomodulator is proven to be more effective than anti-TNF monotherapy, and patients who lose response to an anti-TNF agent should undergo dose intensification in order to regain clinical response. Despite therapeutic optimization, a significant percentage of patients will not achieve clinical remission with anti-TNF agents, and so newer therapies are on the horizon.
\end{abstract}

Keywords: ulcerative colitis, inflammatory bowel disease, infliximab, adalimumab, golimumab

\section{Introduction}

Ulcerative colitis (UC), one subtype of inflammatory bowel disease (IBD), is a chronic inflammatory condition of the colonic mucosa. Patients may suffer from symptoms such as abdominal pain and bloody diarrhea, and endoscopic findings include erythema, edema, and ulcerations in a continuous pattern extending proximally from the rectum, sometimes involving the entire colon. Disease severity can range from mild inflammation in patients with few symptoms to severe fulminant UC that can progress to a surgical emergency, such as toxic megacolon or perforation. Long-term complications of UC can include colonic strictures, colonic dysplasia, and colorectal cancer. ${ }^{1}$

Treatment options for UC have rapidly expanded over the past 10 years and now include multiple biologic agents in addition to prior medication options, such as 5-aminosalicylates, immunomodulators, and corticosteroids. The goals of treatment include resolution of gastrointestinal symptoms, healing of the colonic mucosa, prevention of long-term disease complications, as well as improvement of extra-intestinal symptoms. ${ }^{1}$
Harborview Medical Center, 325 Ninth

Avenue, Box 359773, Seattle, WA, USA

Tel +l 2067447055

Fax +I 2067448698

Email anitaa@medicine.washington.edu
Therapeutics and Clinical Risk Management 2015:II 63-73 
Surgery, typically a total colectomy, is also a potential treatment option. This is not without risks, including pouch failure, pelvic sepsis, infertility in women, and nocturnal fecal soiling. ${ }^{2}$ Therefore, surgery is reserved for patients with severe refractory disease, surgical emergencies, or patients with colorectal dysplasia or cancer. In a cohort of 1,200 patients, the probability of colectomy within the first 5 years of diagnosis was $9 \%$ for patients with distal colitis and $35 \%$ for patients with pan-colitis, most often due to failed medical therapy. ${ }^{3}$ In a more recent population-based study of patients with UC in Manitoba, the rate of colectomy after 20 years of disease was $14.8 \%$, suggesting this rate has decreased over time. ${ }^{4}$ Even after colectomy, patients may have ongoing trouble with inflammation. For example, the rate of pouchitis after an ileal pouch anal anastomosis ranges from $15.5 \%$ after 1 year to $45.5 \%$ after 10 years. $^{5}$

\section{Current and emerging anti-TNF therapies}

It is important to optimize our medical therapies in order to prevent the need for surgical intervention and other complications of disease progression. Select tumor necrosis factoralpha (TNF- $\alpha$ ) antagonists are currently US Food and Drug Administration (FDA) approved for treatment of patients with moderate to severe UC who have had an inadequate response to conventional therapies.

TNF- $\alpha$ is a pro-inflammatory and immunoregulatory cytokine that enhances leukocyte migration, promotes transcription of several inflammatory genes, and causes apoptosis of intestinal epithelial cells, although its exact mechanism of action in IBD is unknown. ${ }^{6}$ It is present in increased concentrations in the serum, colon, and stool of patients with UC. ${ }^{7-9}$ Three TNF- $\alpha$ inhibitors currently approved for the treatment of moderate to severe UC are infliximab, adalimumab, and golimumab. These agents bind TNF- $\alpha$, neutralize its activity, and prevent it from binding to its receptor. Infliximab and adalimumab have also been shown to induce apoptosis of activated $\mathrm{T}$ cells and macrophages. ${ }^{10,11}$

\section{Infliximab}

Infliximab, the first biologic therapy approved for use in UC, is a mouse-human chimeric immunoglobulin $\mathrm{G}$ (IgG) 1 monoclonal antibody to TNF- $\alpha$. It was first studied in Crohn's disease and found to be effective for inducing and maintaining clinical remission, closing fistulas, and sparing corticosteroids. ${ }^{12,13}$ The use of infliximab in UC was initially investigated in several small open-label studies. In 2001 , a series of 16 patients with severe refractory UC were treated with one or two infliximab infusions; $88 \%$ of patients experienced clinical, endoscopic, and histologic improvement, while surgery was avoided in $86 \%$ of patients. ${ }^{14}$ Another pilot study of infliximab in patients with steroid refractory UC showed treatment success at 2 weeks in four of eight patients who received infliximab, compared with zero of three patients who received placebo. ${ }^{15}$ A subsequent retrospective study of 27 patients who received various dosing intervals of infliximab, most receiving only one infusion, showed $44 \%$ clinical remission and $66 \%$ clinical response. ${ }^{16}$

Based on initial promising results, two randomized, double blind placebo controlled studies were initiated to evaluate the efficacy of infliximab as induction and maintenance therapy in UC: The Active Ulcerative Colitis Trials 1 and 2 (ACT 1 and ACT 2). In each study, 364 patients with moderate-severe UC despite treatment with, or intolerance to, corticosteroids were enrolled (Tables 1 and 2). Concurrent medications were held steady throughout the trial, except for prednisone, which was gradually tapered. Approximately one half of patients were on concurrent corticosteroids, three quarters on concurrent 5-aminosalicylates, and one half on concurrent thiopurines. ${ }^{17}$

In both the induction and maintenance arms of these trials, there was a significantly greater rate of clinical response and remission in patients receiving infliximab compared with those receiving placebo (Figures 1-4), and there was no increased benefit of the infliximab $10 \mathrm{mg} / \mathrm{kg}$ dosing compared with $5 \mathrm{mg} / \mathrm{kg}$ dosing. In the ACT 1 trial, mucosal healing at week 8 was significantly higher in patients receiving infliximab: $62 \%$ in the infliximab $5 \mathrm{mg} / \mathrm{kg}$ group and $34 \%$ in the placebo group $(P<0.001)$. Rates were similar in the ACT 2 trial. Differences were maintained, but with less robust response rates at weeks 30 and 54. In both studies, an increased proportion of patients attained corticosteroidfree remission in the infliximab-treated groups, although this percentage was only approximately $20 \%-25 \%$ at week 30. There was no overall increased risk of adverse events in the infliximab group, but there was a slightly increased number of lupus-like reactions (one patient) and neurologic diseases (three patients). In ACT 1, there was an increased risk of infections requiring antibiotics in those receiving infliximab (32\% versus [vs] 21\%,P=0.01). In ACT 2, the percentage of patients with serious adverse events requiring study discontinuation was higher in the placebo group $(10 \%$ vs $2 \%-4 \%, P=0.01) .{ }^{17}$

In an analysis of quality of life scores, patients with UC had a lower health related quality of life at baseline 
Table I Definition of terms

\begin{tabular}{|c|c|}
\hline Term & Definition \\
\hline Moderate - severe ulcerative colitis & Mayo score 6-12 (range 0-12) \\
\hline Partial Mayo score & Mayo score without endoscopic sub-score (range 0-9) \\
\hline Clinical response & $\begin{array}{l}\text { Decrease from baseline of } \geq 3 \text { points in total Mayo score, and at } \\
\text { least } 30 \% \text {, with decrease in rectal bleeding sub-score of } \geq 1 \text { point } \\
\text { (or an absolute sub-score of } 0 \text { or } I \text { ) }\end{array}$ \\
\hline Clinical remission & Total Mayo score of $\leq 2$, with no individual sub-score $>$ I \\
\hline Mucosal healing & Endoscopy sub-score of 0 or 1 \\
\hline Clinical flare & $\begin{array}{l}\text { Increase from baseline in partial Mayo score of } \geq 2 \text { points with } \\
\text { absolute partial Mayo score } \geq 4 \text {, or absolute partial Mayo score } \geq 7\end{array}$ \\
\hline
\end{tabular}

compared with the general population. After treatment, patients receiving infliximab had a significant improvement in Inflammatory Bowel Disease Questionnaire (IBDQ) total score at week 8 , compared with placebo. Continued benefit was seen at weeks 30 and $54 .{ }^{18}$ Additionally, further analysis of patients from the ACT 1 and ACT 2 trials showed a $7 \%$ absolute reduction in the risk of colectomy through 54 weeks of treatment with infliximab, compared with placebo $(P=0.02)$. Factors associated with an increased risk of colectomy were corticosteroid use, elevated C-reactive protein (CRP), Mayo score $\geq 10$, and duration of disease $<3$ years. Patients treated with infliximab also had fewer UC-related hospitalizations $(P=0.003) .{ }^{19}$

In patients who have an inadequate initial response and those who lose response over time, infliximab dosing can be intensified by increasing the administered dose or shortening the dosing interval, in order to regain clinical response. This approach has been proven effective in patients with Crohn's disease. ${ }^{20}$ Additionally, detectable infliximab trough levels

Table 2 Mayo scoring system for assessment of ulcerative colitis activity

\begin{tabular}{|c|c|}
\hline Measure & Scoring system \\
\hline $\begin{array}{l}\text { Stool frequency } \\
\text { (per day) }\end{array}$ & $\begin{array}{l}0=\text { normal number of stools for patient } \\
1=1-2 \text { more stools than normal } \\
2=3-4 \text { more stools than normal } \\
3=5+\text { more stools than normal }\end{array}$ \\
\hline Rectal bleeding & $\begin{array}{l}0=\text { no blood seen } \\
1=\text { streaks of blood with stool less than } 50 \% \text { of time } \\
2=\text { obvious blood with stool most of time } \\
3=\text { passes blood without stool }\end{array}$ \\
\hline $\begin{array}{l}\text { Findings on } \\
\text { endoscopy }\end{array}$ & $\begin{array}{l}0=\text { normal or inactive disease } \\
1=\text { mild disease } \\
2=\text { moderate disease } \\
3=\text { severe disease }\end{array}$ \\
\hline $\begin{array}{l}\text { Physician's global } \\
\text { assessment }\end{array}$ & $\begin{array}{l}0=\text { normal } \\
1=\text { mild disease } \\
2=\text { moderate disease } \\
3=\text { severe disease }\end{array}$ \\
\hline
\end{tabular}

are associated with higher rates of clinical and endoscopic remission in $\mathrm{UC} .{ }^{21}$ In retrospective studies, at least $40 \%$ of patients with UC require dose escalation. ${ }^{22-24}$ In a recent small single center retrospective study, the rate of clinical remission after dose intensification was only $19 \%$ at 12 months. ${ }^{23}$ In contrast, another small single center retrospective review from Japan showed 16 of 17 secondary non-responders were able to achieve clinical remission after infliximab dose intensification..$^{24}$ Another recent retrospective study showed slightly less robust results, as 28 of 41 patients with secondary non-response to infliximab achieved clinical remission at 1 year after dose intensification. ${ }^{25}$ Although these data are fairly limited in UC, dose intensification is recommended in anti-TNF non-responders.

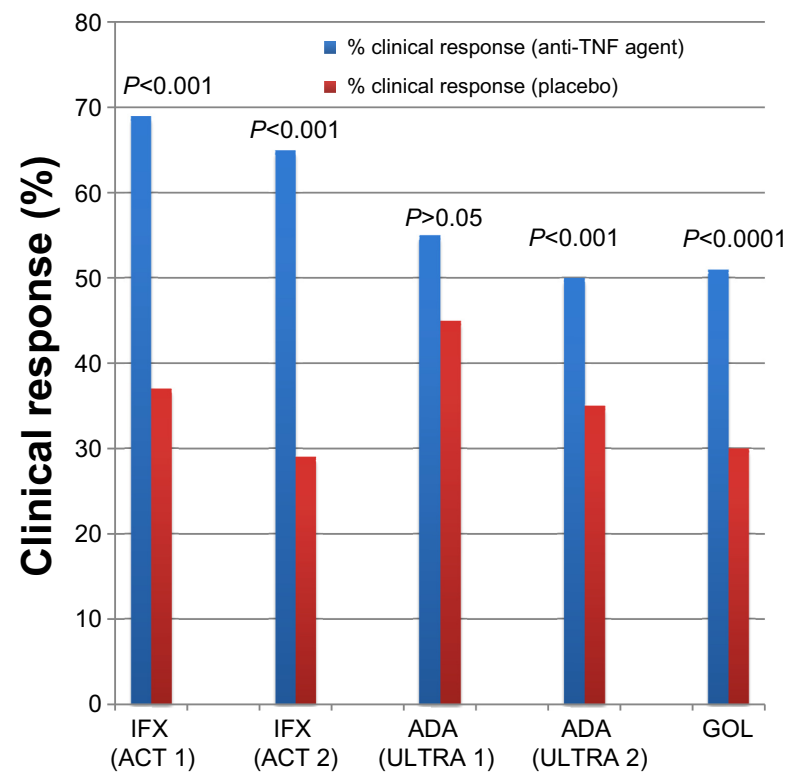

Figure I Anti-TNF agents for induction therapy in ulcerative colitis (clinical response). Notes: There are five separate studies represented on this graph. There are no head-to-head comparisons of these medications, and any comparisons among studies should be undertaken with caution, since studies were performed at different times, with different protocols, and with different patient populations.

Abbreviations: TNF, tumor necrosis factor; IFX, infliximab; ACT, Active Ulcerative Colitis Trials; ADA, adalimumab; ULTRA, Ulcerative Colitis Long-Term Remission and Maintenance with Adalimumab; GOL, golimumab. 


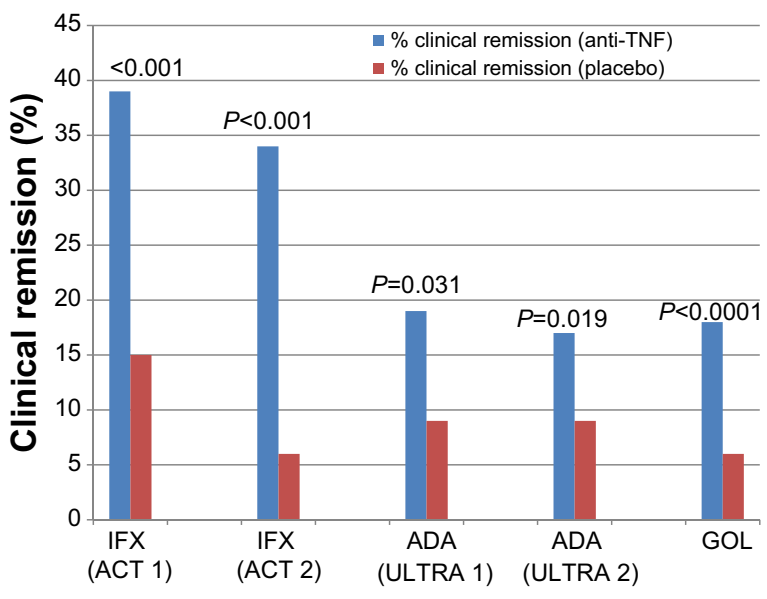

Figure 2 Anti-TNF agents for induction therapy in ulcerative colitis (clinical remission). Notes: There are five separate studies represented on this graph. There are no head-to-head comparisons of these medications, and any comparisons among studies should be undertaken with caution, since studies were performed at different times, with different protocols, and with different patient populations.

Abbreviations: TNF, tumor necrosis factor; IFX, infliximab; ACT, Active Ulcerative Colitis Trials; ADA, adalimumab; ULTRA, Ulcerative Colitis Long-Term Remission and Maintenance with Adalimumab; GOL, golimumab.

Infliximab is also used as rescue therapy for patients with severe acute steroid refractory UC. A 2005 randomized double blind placebo controlled study of infliximab vs placebo in 45 patients showed a significantly increased risk of colectomy in patients treated with placebo (odds ratio [OR] 4.9; 95\% confidence interval [CI] 1.4-17). ${ }^{26}$ Because cyclosporine has traditionally been the preferred treatment for patients with severe steroid refractory UC, numerous

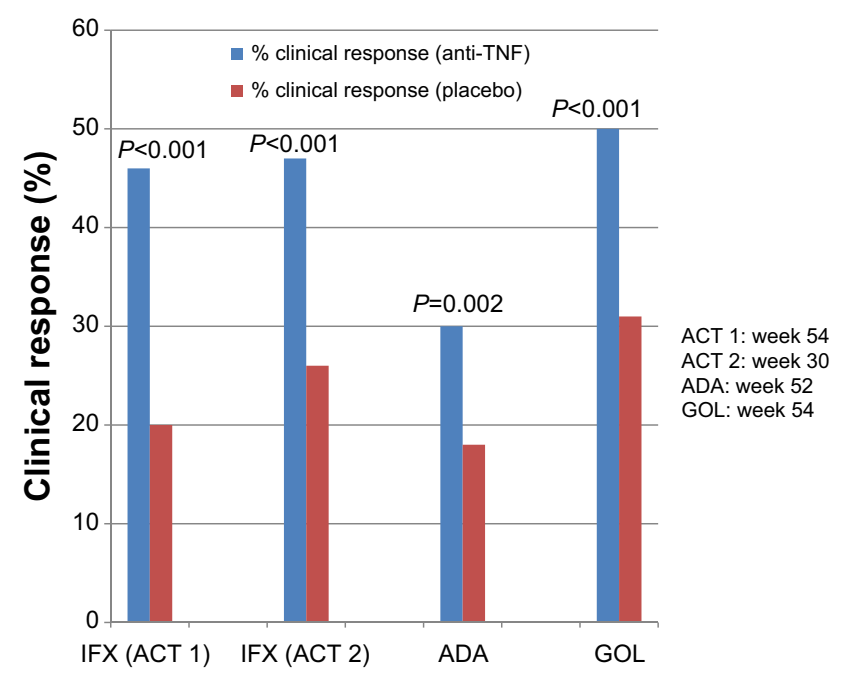

Figure 3 Anti-TNF agents for maintenance therapy in ulcerative colitis (clinical response).

Notes: There are four separate studies represented on this graph. There are no head-to-head comparisons of these medications, and any comparisons among studies should be undertaken with caution, since studies were performed at different times, with different protocols, and with different patient populations.

Abbreviations: TNF, tumor necrosis factor; IFX, infliximab; ACT, Active Ulcerative Colitis Trials; ADA, adalimumab; GOL, golimumab.

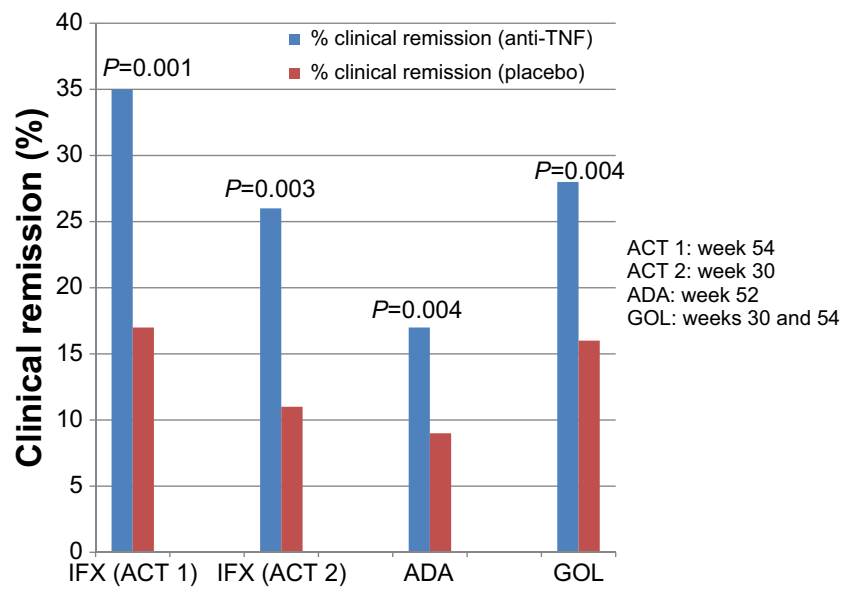

Figure 4 Anti-TNF agents for maintenance therapy in ulcerative colitis (clinical remission).

Notes: There are four separate studies represented on this graph. There are no head-to-head comparisons of these medications, and any comparisons among studies should be undertaken with caution, since studies were performed at different times, with different protocols, and with different patient populations.

Abbreviations: TNF, tumor necrosis factor; IFX, infliximab; ACT, Active Ulcerative Colitis Trials; ADA, adalimumab; GOL, golimumab.

studies have compared cyclosporine to infliximab. A recent retrospective review of 50 patients hospitalized for steroid refractory UC showed that patients treated with cyclosporine and infliximab had similar rates of colectomy $(-30 \%)$ over a 2-8-year follow-up. ${ }^{27} \mathrm{~A}$ prospective non-randomized study of 83 patients with acute severe UC showed the colectomy-free rate was $76 \%$ at 3 months and $65 \%$ at 12 months for patients who received infliximab, significantly better than those receiving cyclosporine $(P=0.04)$. Additionally, there were fewer serious adverse events in patients receiving infliximab. ${ }^{28} \mathrm{~A}$ randomized clinical trial showed a similar rate of treatment failure at day $98(54 \%-60 \%)$ in patients with severe steroid refractory disease receiving infliximab or cyclosporine, with similar rates of adverse events. ${ }^{29}$ In this study, there was no dose escalation of infliximab for patients with an inadequate response, perhaps underestimating its efficacy. In fact, a recent retrospective study showed that an accelerated infliximab dosing regimen (three doses within a median period of 24 days) reduced the rate of colectomy to $7 \%$ during induction therapy, compared with $40 \%$ of patients receiving standard infliximab dosing $(P=0.039){ }^{30}$

\section{Adalimumab}

Adalimumab is a subcutaneously administered recombinant fully human monoclonal antibody against TNF- $\alpha$. Initial small open label studies suggested its efficacy in inducing remission in patients with $\mathrm{UC}$, including patients with prior anti-TNF exposure. ${ }^{31,32}$ The Ulcerative Colitis Long-Term Remission and Maintenance with Adalimumab (ULTRA) 1 
trial included 576 patients with moderate-severe UC failing treatment with, or intolerant to, oral corticosteroids and/or immunomodulators. Patients were divided into three groups: placebo, adalimumab $160 \mathrm{mg} / 80 \mathrm{mg}$ induction dosing, and adalimumab $80 \mathrm{mg} / 40 \mathrm{mg}$ induction dosing. The majority of patients were on concurrent corticosteroids and/or immunomodulators. Patients receiving the higher adalimumab dose had a statistically greater rate of clinical remission and a numerically higher rate of clinical response at week 8 , compared with placebo (Figures 1 and 2). There was also a slightly higher rate of mucosal healing (46.9\% adalimumab $160 / 80$ vs $41.5 \%$ placebo) at week 8 in the adalimumab arm, but this was not statistically significant. Placebo responses were surprisingly high: $44.6 \%$ clinical response and $41.5 \%$ mucosal healing. Patients with higher Mayo scores, higher CRP, and higher baseline weight had reduced remission rates, suggesting that patients with more severe disease have lower rates of response, perhaps due to increased clearance of the medication. Additionally, the weight-independent single dosing schedule may be suboptimal in patients with higher body mass indices. Rates of adverse events were similar across all groups. ${ }^{33}$ Since there was no plateau in dose response, it is unknown if higher induction doses may be even more effective.

ULTRA 2, the trial of maintenance adalimumab, enrolled similar patients except $40 \%$ of these patients had prior treatment with an anti-TNF agent. In this study, patients treated with adalimumab had higher rates of clinical response and remission at weeks 8 and 52 (Figures 3 and 4), as well as greater rates of mucosal healing at week $8(41.1 \%$ vs $31.7 \%$; $P=0.032)$ and week $52(25 \%$ vs $15.4 \% ; P=0.009)$. Adalimumab drug levels were higher in those who achieved remission. ${ }^{34}$ After 1 year of treatment, $13.3 \%$ of patients receiving adalimumab achieved steroid-free remission, compared with $5.7 \%$ receiving placebo $(P=0.035)$. In patients with prior anti-TNF exposure, there was no difference between adalimumab and placebo in the percentage achieving clinical remission at week 8 , but there was a significant difference at week $52(10.2 \%$ vs $3 \% ; P=0.039) .{ }^{34}$ Additional information regarding the use of adalimumab in patients with prior exposure to infliximab comes from a retrospective study of 30 patients who received compassionate use adalimumab after failure of infliximab. In this study, $60 \%$ of patients achieved clinical response at 12 weeks. ${ }^{35}$ Of the patients tested for adalimumab antibodies in ULTRA 2, the $2.9 \%$ who had detectable antibodies were receiving adalimumab monotherapy. Overall, patients receiving adalimumab had lower rates of hospitalization for any cause. ${ }^{36}$
A recent post hoc analysis of the ULTRA 2 trial investigated the use of weekly adalimumab dosing in patients with primary or secondary lack of response to bi-weekly dosing. In primary adalimumab responders who subsequently lost response, weekly adalimumab dosing was able to regain clinical response and mucosal healing in $45 \%$ of patients. In those without an initial response to adalimumab, weekly adalimumab dosing achieved 25\% clinical response and $29 \%$ mucosal healing at week $52 .{ }^{37}$ This evidence supports the strategy of anti-TNF dose escalation in primary and secondary non-responders, and is consistent with data showing increased rates of clinical response in patients with higher adalimumab levels, although it is important to consider that patients with more severe disease may have increased drug clearance due to protein loss from colonic inflammation.

\section{Golimumab}

The most recent anti-TNF agent, golimumab, is a subcutaneously administered transgenic fully human monoclonal IgG1 TNF $\alpha$ antibody that is also approved for the treatment of rheumatologic conditions. It differs from other TNF $\alpha$ antibodies with regard to its affinity for TNF $\alpha$ and protein stability, and it targets a unique epitope on the TNF $\alpha$ molecule. In vitro studies have shown that the drug's affinity for soluble and transmembrane TNF $\alpha$ is superior to that of infliximab and adalimumab, as is its ability to neutralize TNF $\alpha{ }^{38}$

Patients enrolled in the Program of Ulcerative Colitis Research Studies Utilizing an Investigational Treatment-Subcutaneous (PURSUIT-SC) trial of golimumab had moderate-severe disease despite prior treatment with at least one medication for UC, excluding anti-TNF agents or anti-integrin therapies. In the phase 3 trial, 774 patients were randomized to receive placebo, golimumab 200/100 induction dosing, or golimumab 400/200 induction dosing. Median duration of UC was 4.2 years; $40 \%$ of patients had severe disease with a Mayo endoscopic sub-score of 3, and $42 \%$ were reported to have extensive disease. The majority of patients were on 5-aminosalicyates, with approximately half on corticosteroids, and one third on immunomodulators. ${ }^{39}$

Results at week 6 showed a significantly greater rate of clinical response and clinical remission in patients receiving golimumab (Figures 1 and 2), as well as an increased rate of mucosal healing (42\% in golimumab 200/100 mg group) compared to placebo $(29 \%)(P=0.0014)$. Patients receiving golimumab had a greater reduction in IBDQ score (15 vs 27 points; $P<0.0001$ ), showing an improvement in healthrelated quality of life. As early as week 2 , golimumab-treated patients were noted to have an improvement in partial 
Mayo score and CRP level. Patients in the highest quartile of golimumab serum levels had the greatest decrease in Mayo score, and the highest percentage of clinical response and remission. Females and Caucasians were more likely to achieve clinical response or remission, as well as patients on corticosteroids and those with lower baseline fecal lactoferrin, CRP, and Mayo scores. Efficacy results at week 6 were similar between the two golimumab dosing regimens. ${ }^{39}$

There were similar proportions of patients with total adverse events, serious adverse events, and serious infections across all groups. One patient on golimumab, also on concurrent prednisolone, died from peritonitis after surgical complications related to an ischiorectal abscess. Another patient on golimumab developed a demyelinating disorder after completing induction and being randomized to placebo in the maintenance study. There were no serious injection site reactions reported. ${ }^{39}$

After the 6 week induction study, responders were randomized to placebo or continued golimumab 50 or $100 \mathrm{mg}$ every 4 weeks through week 52. Patients who failed to respond in the induction study were given open-label golimumab $100 \mathrm{mg}$ every 4 weeks. Patients who responded to induction therapy but subsequently lost response underwent modifications to their therapies: patients receiving placebo were switched to golimumab $100 \mathrm{mg}$ dosing, and patients receiving $50 \mathrm{mg}$ golimumab were re-randomized to receive golimumab $50 \mathrm{mg}$ or $100 \mathrm{mg}$ every 4 weeks. There were 464 randomized patients included in the efficacy analyses and 764 nonrandomized patients included in pharmacokinetic and safety analyses. At the beginning of the maintenance trial, approximately $50 \%$ of patients were receiving corticosteroids, $80 \%$ were receiving 5 -aminosalicylates, and $30 \%$ were receiving thiopurines. ${ }^{40}$

A significantly greater percentage of patients receiving golimumab achieved clinical response (number needed to treat 5) and remission (number needed to treat 8 ) at week 54, compared with the placebo group (Figures 3 and 4). Factors associated with increased likelihood of clinical remission included lower baseline Mayo score, lower baseline fecal lactoferrin, and higher baseline albumin level, suggesting that patients with less severe disease had a greater likelihood of response. In an analysis of mucosal healing at both weeks 30 and $54,42.4 \%$ of patients receiving golimumab $100 \mathrm{mg}$ achieved this result, compared to only $26.6 \%$ of placebo arm $(P=0.002)$. At week 54, 23.2\% of the golimumab $100 \mathrm{mg}$ group and $18.4 \%$ of the placebo arm achieved corticosteroidfree remission $(P=0.423) .{ }^{40}$ In summary, absolute response rates with golimumab were: $53 \%$ induction of clinical response, $18 \%$ induction of clinical remission, $25 \%$ clinical response at 1 year, 17\% clinical remission at 1 year, and $20 \%$ mucosal healing at 1 year. ${ }^{41}$ Since patients with prior anti-TNF exposure were excluded from this study, it is not clear if golimumab will be effective for prior anti-TNF non-responders.

Serum golimumab concentrations were measured throughout the trial, and steady state drug levels were reached at week 8. Patients with serum golimumab concentrations in the higher quartiles achieved greater rates of clinical response and remission compared with those patients in the lower quartiles. Antibodies to golimumab were rare but more common in patients not receiving concomitant immunomodulators $(3.8 \%$ vs $1.1 \% ; P=0.013) .{ }^{40}$ The fact that patients with higher golimumab levels achieved greater rates of response, and those with less severe disease (lower CRP, lower Mayo score, lower fecal lactoferrin) also had a greater response, raises the question of whether the lower response rates in patients with more severe disease could be due to increased drug clearance. It is possible that doseescalation of golimumab could be effective in these patients with lower drug levels, although it is noted that there was no difference in the rate of clinical response in secondary non-responders who received dose escalation in this study, compared to those who maintained the $50 \mathrm{mg}$ dose. ${ }^{40}$ This was a relatively small number of patients, and further studies are needed to evaluate this. ${ }^{41}$

In the golimumab maintenance trial, the incidence of adverse events across treatment groups was similar. However, the proportion of patients who experienced a serious adverse event or discontinued treatment due to an adverse event was higher in the golimumab $100 \mathrm{mg}$ group compared with the $50 \mathrm{mg}$ and placebo groups. The duration of follow-up for placebo patients was shorter, and when controlled for this, rates of adverse events leading to discontinuation were comparable, and rates of serious adverse events were less discrepant. Injection site reactions were more likely in patients receiving golimumab $100 \mathrm{mg}(7.1 \%)$ compared with $50 \mathrm{mg}$ $(1.9 \%)$ and placebo $(1.9 \%) .{ }^{40}$

There were three deaths reported through week 54 of the trial, all in the golimumab $100 \mathrm{mg}$ group. Causes of death were malnutrition in one patient, cardiac failure in one patient, and disseminated tuberculosis (TB) in a patient who tested positive for latent TB on study entry and was receiving isoniazid. There were six deaths reported after week 54, five of them in patients who received golimumab: biventricular heart failure in a patient with marked atherosclerosis, myocardial infarction in a patient with a history of myocardial 
infarction, metastatic gallbladder adenocarcinoma, sepsis, and accidental nitrous oxide overdose. TB was found in four patients, all from endemic areas and on concurrent corticosteroid therapy before entry into study, three of whom remained on corticosteroids at the time of diagnosis. Two patients receiving golimumab developed severe infections (cytomegalovirus and brain abscess). ${ }^{40}$

\section{Combination therapy with azathioprine}

Another important question in UC treatment is the benefit of combination therapy. The recent UC-SUCCESS trial of patients with moderate-severe disease who had a prior inadequate response to corticosteroids investigated the use of combination therapy with infliximab and azathioprine, compared with infliximab or azathioprine alone. This study of 239 patients showed a significant improvement in the percentage of patients achieving corticosteroid-free remission at week 16 in the combination therapy group compared with either therapy alone $(39.7 \%$ combination, $23.7 \%$ azathioprine, $22.1 \%$ infliximab; $P=0.017$ combination vs infliximab, $P=0.032$ combination vs azathioprine). Mucosal healing was achieved in $63 \%$ of patients on combination therapy, compared with $55 \%$ of patients receiving infliximab monotherapy and $37 \%$ of those receiving azathioprine monotherapy ( $P=0.295$ combination vs infliximab, $P=0.001$ combination vs azathioprine). ${ }^{42}$ These data are consistent with evidence in Crohn's disease showing increased efficacy of combination therapy compared to anti-TNF monotherapy..$^{43}$ Unfortunately, the study was stopped early, so there are no data on combination therapy for maintenance in UC. ${ }^{44}$ Nonetheless, this study provides important information showing that combination therapy for induction of remission in UC is superior to either therapy alone. In patients without contraindications to either class of medications, combination therapy should be strongly considered after counseling regarding the increased risk of lymphoma and skin cancer in patients on thiopurines.

\section{Efficacy, safety, and tolerability of anti-TNF therapy}

All three TNF inhibitors carry potential risks associated with their use, and should be avoided in patients with uncontrolled infections, advanced heart failure (New York Heart Association Class III or IV), and neurologic conditions, specifically demyelinating diseases. Demyelinating disorders are rarely seen in patients receiving anti-TNF agents, and a causal relationship has not been definitively established, but it is recommended to avoid these agents in patients with a history of such conditions. Additionally, the use of anti-TNF agents is associated with increased mortality in patients with advanced heart failure. Prior to initiating anti-TNF treatment, patients should be screened for TB and Hepatitis B virus, and appropriately treated for these conditions if tests return positive. It is also important to ensure that patients are up to date on immunizations, particularly those against influenza, pneumococcal infection, and human papillomavirus infection. Live vaccines are contraindicated while patients are on anti-TNF therapy and for 3 months after stopping therapy. Anti-TNF agents should be used with caution in patients with a history of malignancy, due to a potential risk of accelerating the growth of a tumor. ${ }^{45,46}$

Other potential adverse effects of anti-TNF therapy include acute infusion reactions, which occur in approximately $10 \%$ of patients treated with infliximab, and serious infusion reactions including anaphylaxis, convulsions, and hypotension, which occur in approximately $1 \%$. Injection site reactions and rare anaphylactic reactions can also occur with subcutaneously administered anti-TNF agents. Other possible adverse effects include neutropenia, hepatotoxicity, serum sickness, leukocytoclastic vasculitis, rash including psoriasiform rash, and induction of autoimmunity. ${ }^{45}$ Approximately $50 \%$ of patients receiving infliximab develop antinuclear antibodies after 2 years, but drug-induced lupus is rare. ${ }^{47}$ If a patient develops a psoriasiform rash, this almost always resolves with cessation of the anti-TNF agent. If another anti-TNF agent is initiated, the risk of recurrence is approximately $50 \%{ }^{48}$

Serious infections occur in $2 \%-4 \%$ of patients treated with TNF antibodies. In a 5-year follow-up of the TREAT (Crohn's Therapy, Resource, Evaluation, and Assessment Tool) registry, a large observational study of patients with Crohn's disease, infliximab was associated with an increased risk of serious infection (hazard ratio [HR] 1.43; CI 1.111.84); however, risks were greater for patients treated with narcotics (HR 1.98; CI 1.44-2.73) and prednisone (HR 1.57; CI 1.17-2.10), and for those with moderate-severe disease activity (HR 2.24; CI 1.57-3.19). ${ }^{49}$ The use of combination therapy with two immunosuppressive medications (including steroids, immunomodulators and/or anti-TNF agents) is associated with significantly increased rates of infections, including TB, candidiasis, herpes zoster, and sepsis. ${ }^{50}$ A 2011 meta-analysis of all randomized controlled trials and prospective cohorts of anti-TNF agents in IBD, rheumatoid arthritis, and psoriasis showed a small increased risk of serious infections (OR 1.37; CI 1.04-1.82). The risk was not statistically increased when evaluating studies with IBD patients alone (OR 1.28; CI 0.67-2.44). The time of highest risk was the first 
6 months after initiation of therapy. The risk was higher with infliximab (OR 1.97; CI 1.41-2.75) and certolizumab (OR 2.82; CI 1.27-6.29), although certolizumab is not approved for use in UC. . $^{51,52}$

In contrast, a meta-analysis of patients with Crohn's disease on anti-TNF therapy did not show an increased risk of serious infections requiring antimicrobial therapy or hospitalization. ${ }^{53}$ A recent comparative safety meta-analysis of 11,700 patients with rheumatoid arthritis receiving antiTNF agents showed the overall rate of serious adverse events in patients treated with anti-TNFs was not statistically different from those treated with placebo and/or traditional diseasemodifying antirheumatic drug, and there was no statistical difference in the risk of malignancy. There was, however, an increased risk of serious infection in patients receiving TNF inhibitors (OR 1.42; CI 1.13-1.78), specifically true of infliximab (OR 1.63; CI 1.07-2.47) and adalimumab (OR 1.69; CI 1.12-2.54). Golimumab also had a numeric increased risk of serious infection (OR 1.55; CI 0.76-3.17), but this was not statistically significant. ${ }^{54}$ Additionally, a 5-year study of golimumab in patients with psoriatic arthritis showed a similar safety profile to other anti-TNF agents. ${ }^{55}$ Overall, it appears the risk of serious infection with anti-TNF agents is relatively small, and may be modified by concurrent therapies and disease severity. Regardless of the magnitude of the risk, it is important to employ preventative measures, including appropriate vaccination and pre-treatment testing for TB and Hepatitis B virus. ${ }^{51}$

There has also been concern for a possible increased risk of malignancy in patients on anti-TNF therapy, specifically lymphoma. Based on recent studies, the risk of lymphoma seems to derive primarily from the thiopurines used in combination therapy. ${ }^{56-58}$ Patients on anti-TNF therapy may have a small increased risk of both melanoma and non-melanoma skin cancers, and so should be advised on the importance of sun protection and regular skin examinations. ${ }^{51,59,60}$

There have been no head-to-head trials comparing the efficacy of anti-TNF agents in UC. Using data from the trials previously described, there have been three indirect treatment comparison meta-analyses. Thorlund et $\mathrm{al}^{61}$ performed a comparison between infliximab and adalimumab in anti-TNF naïve patients, using data from the ACT and ULTRA trials. After 8 weeks of induction treatment, rates of clinical response (OR 0.45; CI 0.23-0.89), clinical remission (OR 0.42; CI 0.17-0.97), and mucosal healing (OR 0.46; CI 0.25-0.86) were higher in patients treated with infliximab, compared with those treated with adalimumab. At 52 weeks, there remained a trend toward favoring infliximab, but this was not statistically significant. Infliximab was associated with a higher likelihood of sustained response. There were no differences in adverse outcomes in treatment or placebo groups. ${ }^{61}$

A recent systematic review and network meta-analysis, including 2,282 patients and seven double-blind placebocontrolled trials, showed that all biologic agents (infliximab, adalimumab, golimumab, vedolizumab) increased clinical response, clinical remission, and mucosal healing for induction therapy compared with placebo. ${ }^{62}$ Indirect comparisons suggested that infliximab was more effective than adalimumab in inducing clinical response (OR 2.36; CI 1.22-4.63) and mucosal healing (OR 2.0; CI 1.13-3.59). No other indirect comparison reached statistical significance. The occurrence of adverse events was not different between biologic agents and placebo, but there was a high rate of incomplete follow-up, and so there may have been inadequate ascertainment of harms. ${ }^{62}$ A second similar meta-analysis, also published in 2014, included an additional 2003 study from Probert et al investigating the use of infliximab in steroid refractory $\mathrm{UC} .{ }^{63}$ In this second meta-analysis, there was a trend toward increased efficacy of infliximab compared with adalimumab and golimumab, but this did not reach statistical significance. $^{64}$

\section{Conclusion}

In summary, all three anti-TNF agents are shown to be effective in inducing and maintaining clinical response and remission in patients with $\mathrm{UC}$, with fairly comparable safety profiles. Although indirect comparisons by meta-analysis may favor infliximab over adalimumab, there is not enough evidence to change clinical practice, and a randomized controlled clinical trial comparing these agents would be beneficial. For now, the choice of anti-TNF agent is typically based on the cost of treatment and insurance coverage, the route of administration, and convenience and preference of each patient. Golimumab is an additional subcutaneous option for patients with moderate-severe UC, although it has not been studied in patients who have failed to respond to other anti-TNF medications. Regardless of the anti-TNF agent chosen, combination therapy with azathioprine is likely more effective for inducing remission than anti-TNF monotherapy, and should be recommended for patients without thiopurine intolerance or contraindications to its use. Unfortunately, there are still up to $50 \%$ of patients receiving anti-TNF agents who fail to respond to induction dosing, and even more patients who lose response to the anti-TNF agent over time. There are limited data on the use of anti-TNF agents 
in prior anti-TNF non-responders aside from the infliximab experienced patients enrolled in ULTRA 2, which showed a slight benefit of adalimumab over placebo at 52 weeks, but no statistically significant benefit at 8 weeks.

Because not all patients respond to anti-TNF agents, researchers continue to investigate other mechanisms for treatment of IBD, including anti-integrin therapies. Vedolizumab, an $\alpha 4 \beta 7$ integrin inhibitor was recently FDA approved for the treatment of UC. In the GEMINI 1 trial, vedolizumab was found to be more effective than placebo for inducing and maintaining clinical response, clinical remission, and mucosal healing. In theory, because of the unique location of the $\alpha 4 \beta 7$ integrin receptors in gastrointestinal mucosa alone, it may be expected that vedolizumab will have a reduced risk of systemic infections and malignancy compared with systemically acting agents. In the GEMINI 1 trial, there was no increased risk of infection, serious adverse reaction, or malignancy in patients receiving vedolizum$a b .{ }^{65}$ Therefore, vedolizumab may be an option for patients with moderate to severe UC who have failed to respond to anti-TNF agents, in those with contraindications to anti-TNF therapy, or in some patients who prefer this medication to anti-TNF agents based on its side effect profile.

\section{Disclosure}

Dr Afzali is a consultant or advisory board physician for Abbvie, UCB, Janssen, and Prometheus Laboratories. Dr Fausel has no conflicts of interest.

\section{References}

1. Osterman MT, Lichtenstein GR. Ulcerative Colitis. In: Sleisenger MH, Feldman M, Friedman LS, Brandt LJ, editors. Sleisenger and Fordtran's gastrointestinal and liver disease pathophysiology, diagnosis, management. 9th ed. Philadelphia, PA: Saunders/Elsevier; 2010: 1975-2013.

2. McIntyre PB, Pemberton JH, Wolff BG, Beart RW, Dozois RR. Comparing functional results one year and ten years after ileal pouchanal anastomosis for chronic ulcerative colitis. Dis Colon Rectum. 1994;37(4):303-307.

3. Langholz E, Munkholm P, Davidsen M, Binder V. Colorectal cancer risk and mortality in patients with ulcerative colitis. Gastroenterology. 1992;103(5):1444-1451.

4. Targownik LE, Singh H, Nugent Z, Bernstein CN. The epidemiology of colectomy in ulcerative colitis: results from a population-based cohort. Am J Gastroenterol. 2012;107(8):1228-1235.

5. Penna C, Dozois R, Tremaine W, et al. Pouchitis after ileal pouch-anal anastomosis for ulcerative colitis occurs with increased frequency in patients with associated primary sclerosing cholangitis. Gut. 1996;38(2):234-239.

6. Billiet T, Rutgeerts P, Ferrante M, Van Assche G, Vermeire S. Targeting TNF-alpha for the treatment of inflammatory bowel disease. Expert Opin Biol Ther. 2014;14(1):75-101.

7. Murch SH, Lamkin VA, Savage MO, Walker-Smith JA, MacDonald TT. Serum concentrations of tumour necrosis factor alpha in childhood chronic inflammatory bowel disease. Gut. 1991;32(8):913-917.
8. Murch SH, Braegger CP, Walker-Smith JA, MacDonald TT. Location of tumour necrosis factor alpha by immunohistochemistry in chronic inflammatory bowel disease. Gut. 1993;34(12):1705-1709.

9. Braegger CP, Nicholls S, Murch SH, Stephens S, MacDonald TT. Tumour necrosis factor alpha in stool as a marker of intestinal inflammation. Lancet. 1992;339(8785):89-91.

10. Scallon BJ, Moore MA, Trinh H, Knight DM, Ghrayeb J. Chimeric antiTNF-alpha monoclonal antibody cA2 binds recombinant transmembrane TNF-alpha and activates immune effector functions. Cytokine. 1995;7(3):251-259.

11. Tracey D, Klareskog L, Sasso EH, Salfeld JG, Tak PP. Tumor necrosis factor antagonist mechanisms of action: a comprehensive review. Pharmacol Ther. 2008;117(2):244-279.

12. Hanauer SB, Feagan BG, Lichtenstein GR, et al. Maintenance infliximab for Crohn's disease: the ACCENT I randomised trial. Lancet. 2002;359(9317):1541-1549.

13. Sands BE, Anderson FH, Bernstein CN, et al. Infliximab maintenance therapy for fistulizing Crohn's disease. $N$ Engl J Med. 2004;350(9): 876-885.

14. Chey WY. Infliximab for patients with refractory ulcerative colitis. Inflamm Bowel Dis. 2001;7 Suppl 1:S30-S33.

15. Sands BE, Tremaine WJ, Sandborn WJ, et al. Infliximab in the treatment of severe, steroid-refractory ulcerative colitis: a pilot study. Inflamm Bowel Dis. 2001;7(2):83-88.

16. Su C, Salzberg BA, Lewis JD, et al. Efficacy of anti-tumor necrosis factor therapy in patients with ulcerative colitis. Am J Gastroenterol. 2002;97(10):2577-2584.

17. Rutgeerts P, Sandborn WJ, Feagan BG, et al. Infliximab for induction and maintenance therapy for ulcerative colitis. $N$ Engl $J$ Med. 2005;353(23):2462-2476.

18. Feagan BG, Reinisch W, Rutgeerts $P$, et al. The effects of infliximab therapy on health-related quality of life in ulcerative colitis patients. Am J Gastroenterol. 2007;102(4):794-802.

19. Sandborn WJ, Rutgeerts P, Feagan BG, et al. Colectomy rate comparison after treatment of ulcerative colitis with placebo or infliximab. Gastroenterology. 2009;137(4):1250-1260.

20. Kopylov U, Mantzaris GJ, Katsanos KH, et al. The efficacy of shortening the dosing interval to once every six weeks in Crohn's patients losing response to maintenance dose of infliximab. Aliment Pharmacol Ther. 2011;33(3):349-357.

21. Seow CH, Newman A, Irwin SP, Steinhart AH, Silverberg MS, Greenberg GR. Trough serum infliximab: a predictive factor of clinical outcome for infliximab treatment in acute ulcerative colitis. Gut. 2010;59(1):49-54.

22. Taxonera C, Olivares D, Mendoza JL, Díaz-Rubio M, Rey E. Need for infliximab dose intensification in Crohn's disease and ulcerative colitis. World J Gastroenterol. 2014;20(27):9170-9177.

23. Rostholder E, Ahmed A, Cheifetz AS, Moss AC. Outcomes after escalation of infliximab therapy in ambulatory patients with moderately active ulcerative colitis. Aliment Pharmacol Ther. 2012;35(5): $562-567$.

24. Yamada S, Yoshino T, Matsuura M, et al. Long-term efficacy of infliximab for refractory ulcerative colitis: results from a single center experience. BMC Gastroenterol. 2014;14:80.

25. Cesarini M, Katsanos K, Papamichael K, et al. Dose optimization is effective in ulcerative colitis patients losing response to infliximab: a collaborative multicentre retrospective study. Dig Liver Dis. 2014;46(2):135-139.

26. Jarnerot G, Hertervig E, Friis-Liby I, et al. Infliximab as rescue therapy in severe to moderately severe ulcerative colitis: a randomized, placebo-controlled study. Gastroenterology. 2005;128(7): $1805-1811$

27. Naves JE, Llao J, Ruiz-Cerulla A, et al. Long-term Comparative Efficacy of Cyclosporine- or Infliximab-based Strategies for the Management of Steroid-refractory Ulcerative Colitis Attacks. Inflamm Bowel Dis. 2014;20(8):1375-1381. 
28. Croft A, Walsh A, Doecke J, Cooley R, Howlett M, Radford-Smith G. Outcomes of salvage therapy for steroid-refractory acute severe ulcerative colitis: ciclosporin vs infliximab. Aliment Pharmacol Ther. 2013;38(3):294-302.

29. Laharie D, Bourreille A, Branche J, et al. Ciclosporin versus infliximab in patients with severe ulcerative colitis refractory to intravenous steroids: a parallel, open-label randomised controlled trial. Lancet. 2012;380(9857):1909-1915.

30. Gibson DJ, Heetun ZS, Redmond CE, et al. An Accelerated Infliximab Induction Regimen Reduces the Need for Early Colectomy in Patients with Acute Severe Ulcerative Colitis. Clin Gastroenterol Hepatol. Epub 2014 Jul 30

31. Afif W, Leighton JA, Hanauer SB, et al. Open-label study of adalimumab in patients with ulcerative colitis including those with prior loss of response or intolerance to infliximab. Inflamm Bowel Dis. 2009;15(9):1302-1307.

32. Peyrin-Biroulet L, Laclotte C, Roblin X, Bigard MA. Adalimumab induction therapy for ulcerative colitis with intolerance or lost response to infliximab: an open-label study. World J Gastroenterol. 2007;13(16):2328-2332.

33. Reinisch W, Sandborn WJ, Hommes DW, et al. Adalimumab for induction of clinical remission in moderately to severely active ulcerative colitis: results of a randomised controlled trial. Gut. 2011;60(6):780-787.

34. Sandborn WJ, van Assche G, Reinisch W, et al. Adalimumab induces and maintains clinical remission in patients with moderate-to-severe ulcerative colitis. Gastroenterology. 2012;142(2):257-265.

35. Taxonera C, Estelles J, Fernandez-Blanco I, et al. Adalimumab induction and maintenance therapy for patients with ulcerative colitis previously treated with infliximab. Aliment Pharmacol Ther. 2011;33(3):340-348.

36. Feagan BG, Sandborn WJ, Lazar A, et al. Adalimumab therapy is associated with reduced risk of hospitalization in patients with ulcerative colitis. Gastroenterology. 2014;146(1):110-118.

37. Wolf D, D'Haens G, Sandborn WJ, et al. Escalation to weekly dosing recaptures response in adalimumab-treated patients with moderately to severely active ulcerative colitis. Aliment Pharmacol Ther. 2014;40(5):486-497.

38. Shealy DJ, Cai A, Staquet K, et al. Characterization of golimumab, a human monoclonal antibody specific for human tumor necrosis factor alpha. MAbs. 2010;2(4):428-439.

39. Sandborn WJ, Feagan BG, Marano C, et al. Subcutaneous golimumab induces clinical response and remission in patients with moderate-tosevere ulcerative colitis. Gastroenterology. 2014;146(1):85-95.

40. Sandborn WJ, Feagan BG, Marano C, et al. Subcutaneous golimumab maintains clinical response in patients with moderate-to-severe ulcerative colitis. Gastroenterology. 2014;146(1):96-109.

41. Hanauer SB. Still in pursuit. Gastroenterology. 2014;146(1):13-15.

42. Panaccione R, Ghosh S, Middleton S, et al. Combination therapy with infliximab and azathioprine is superior to monotherapy with either agent in ulcerative colitis. Gastroenterology. 2014;146(2):392-400.

43. Colombel JF, Sandborn WJ, Reinisch W, et al. Infliximab, azathioprine, or combination therapy for Crohn's disease. $N$ Engl J Med. 2010;362(15):1383-1395.

44. Kamath N, Kamath A, Pai CG. Infliximab for Moderate to Severe Ulcerative Colitis: The Jury Isn't in Yet. Gastroenterology. 2014;147(2):544-545.

45. Nielsen $\mathrm{OH}$, Ainsworth MA. Tumor necrosis factor inhibitors for inflammatory bowel disease. N Engl J Med. 2013;369(8):754-762.

46. Sands BE, Siegel CA. Crohn's Disease. In: Sleisenger MH, Feldman M, Friedman LS, Brandt LJ, editors. Sleisenger and Fordtran's gastrointestinal and liver disease pathophysiology, diagnosis, management. 9th ed. Philadelphia, PA: Saunders/Elsevier; 2010:1941-1973.

47. Vermeire S, Noman M, Van Assche G, et al. Autoimmunity associated with anti-tumor necrosis factor alpha treatment in Crohn's disease: a prospective cohort study. Gastroenterology. 2003;125(1):32-39.
48. Cullen G, Kroshinsky D, Cheifetz AS, Korzenik JR. Psoriasis associated with anti-tumour necrosis factor therapy in inflammatory bowel disease: a new series and a review of 120 cases from the literature. Aliment Pharmacol Ther. 2011;34(11-12):1318-1327.

49. Lichtenstein GR, Feagan BG, Cohen RD, et al. Serious infection and mortality in patients with Crohn's disease: more than 5 years of follow-up in the TREAT registry. Am J Gastroenterol. 2012;107(9):1409-1422.

50. Marehbian J, Arrighi HM, Hass S, Tian H, Sandborn WJ. Adverse events associated with common therapy regimens for moderate-to-severe Crohn's disease. Am J Gastroenterol. 2009;104(10):2524-2533.

51. Targownik LE, Bernstein CN. Infectious and malignant complications of TNF inhibitor therapy in IBD. Am J Gastroenterol. 2013;108(12):1835-1842.

52. Singh JA, Wells GA, Christensen R, et al. Adverse effects of biologics: a network meta-analysis and Cochrane overview. Cochrane Database Syst Rev. 2011(2):CD008794.

53. Peyrin-Biroulet L, Deltenre P, de Suray N, Branche J, Sandborn WJ, Colombel JF. Efficacy and safety of tumor necrosis factor antagonists in Crohn's disease: meta-analysis of placebo-controlled trials. Clin Gastroenterol Hepatol. 2008;6(6):644-653.

54. Michaud TL, Rho YH, Shamliyan T, Kuntz KM, Choi HK. The Comparative Safety of TNF Inhibitors in Rheumatoid Arthritis - A MetaAnalysis Update of 44 Randomized Controlled Trials. Am J Med. Epub 2014 Jun 17.

55. Kavanaugh A, McInnes IB, Mease P, et al. Clinical efficacy, radiographic and safety findings through 5 years of subcutaneous golimumab treatment in patients with active psoriatic arthritis: results from a long-term extension of a randomised, placebo-controlled trial (the GO-REVEAL study). Ann Rheum Dis. 2014;73(9):1689-1694.

56. Osterman MT, Sandborn WJ, Colombel JF, et al. Increased risk of malignancy with adalimumab combination therapy, compared with monotherapy, for Crohn's disease. Gastroenterology. 2014;146(4):941-949.

57. Siegel CA, Marden SM, Persing SM, Larson RJ, Sands BE. Risk of lymphoma associated with combination anti-tumor necrosis factor and immunomodulator therapy for the treatment of Crohn's disease: a meta-analysis. Clin Gastroenterol Hepatol. 2009;7(8):874-881.

58. Caspersen S, Elkjaer M, Riis L, et al. Infliximab for inflammatory bowel disease in Denmark 1999-2005: clinical outcome and follow-up evaluation of malignancy and mortality. Clin Gastroenterol Hepatol. 2008;6(11):1212-1217.

59. Long MD, Herfarth HH, Pipkin CA, Porter CQ, Sandler RS, Kappelman MD. Increased risk for non-melanoma skin cancer in patients with inflammatory bowel disease. Clin Gastroenterol Hepatol. 2010;8(3):268-274.

60. Long MD, Martin CF, Pipkin CA, Herfarth HH, Sandler RS, Kappelman MD. Risk of melanoma and nonmelanoma skin cancer among patients with inflammatory bowel disease. Gastroenterology. 2012; 143(2):390-399.

61. Thorlund K, Druyts E, Mills EJ, Fedorak RN, Marshall JK. Adalimumab versus infliximab for the treatment of moderate to severe ulcerative colitis in adult patients naive to anti-TNF therapy: an indirect treatment comparison meta-analysis. J Crohns Colitis. 2014;8(7):571-581.

62. Danese S, Fiorino G, Peyrin-Biroulet L, et al. Biological agents for moderately to severely active ulcerative colitis: a systematic review and network meta-analysis. Ann Intern Med. 2014;160(10): 704-711.

63. Probert CS, Hearing SD, Schreiber S, et al. Infliximab in moderately severe glucocorticoid resistant ulcerative colitis: a randomised controlled trial. Gut. 2003;52(7):998-1002.

64. Stidham RW, Lee TC, Higgins PD, et al. Systematic review with network meta-analysis: the efficacy of anti-tumour necrosis factor-alpha agents for the treatment of ulcerative colitis. Aliment Pharmacol Ther. 2014;39(7):660-671.

65. Feagan BG, Rutgeerts P, Sands BE, et al. Vedolizumab as induction and maintenance therapy for ulcerative colitis. $N$ Engl $J$ Med. 2013;369(8):699-710. 
Therapeutics and Clinical Risk Management

Dovepress

\section{Publish your work in this journal}

Therapeutics and Clinical Risk Management is an international, peerreviewed journal of clinical therapeutics and risk management, focusing on concise rapid reporting of clinical studies in all therapeutic areas, outcomes, safety, and programs for the effective, safe, and sustained use of medicines. This journal is indexed on PubMed Central, CAS,

EMBase, Scopus and the Elsevier Bibliographic databases. The manuscript management system is completely online and includes a very quick and fair peer-review system, which is all easy to use. Visit http://www.dovepress.com/testimonials.php to read real quotes from published authors.

Submit your manuscript here: http://www.dovepress.com/therapeutics-and-clinical-risk-management-journal 